\title{
EFFECTIVENESS OF CUSTOMER REFERRAL REWARD PROGRAMS: THE MEDIATING ROLE OF METAPERCEPTION
}

\author{
Dominik Georgi, Frankfurt School of Finance \& Management, Germany \\ Jochen Wirtz, National University of Singapore, Singapore \\ Ping Xiao, National University of Singapore, Singapore \\ Christopher Tang, UCLA, USA
}

\begin{abstract}
Customer referral reward programs (RRPs) are ubiquitous especially in the service sector. Yet, their impact on customer behavior has not gained much attention in the research community until recently. The focus of the present study is to examine the effectiveness of RRPs by analyzing a variety of determinants in a real world business setting, where we combined CRM and survey data of private banking customers of a large European bank.

RRPs are used as a customer acquisition tool that utilizes existing customer relationships. The logic behind such programs is that rewards are offered to current customers (i.e., inductors) in order to incentivize them recommending the firm's products to their friends and colleagues (i.e., inductees). However, anecdotal evidence suggests that there might be undesired side effects of incentivizing recommendation behavior within RRPs. For example, current customers might feel uneasy using such a program as they may be afraid how they are perceived by the inductees. Specifically, inductors may not want to be seen to benefit from an inductee's purchase decision. Such psychological processes are called metaperception. Metaperception is the process of determining how one's behavior influences others' judgment of oneself (Laing, Phillipson \& Lee 1966) and one's behavior (Malloy \& Janowski 1992). We propose that metaperception mediates the link between reward size and recommendation behavior, whereby reward size has a negative effect on metaperception, and thereby a negative indirect effect on recommendation behavior. We explore the role of a number of other variables on metaperception, recommendation behaviors, and the effectiveness of an RRP.

Specifically, we examine the effectiveness of RRPs using two dependent variables: (1) the inductor's (i.e., the recommendation giver's) recommendation behavior, and (2) the inductees' (i.e., the recommendation recipient's) behavior and their perception about the products and services offered by the firm. As determinants of our dependent variables, we explored inductor characteristics (e.g., attractiveness/value of the inductor to the firm; relationship proneness; deal proneness; sociological variables such as the size of the social network of the inductor), variables related to the inductor-inductee relationship (e.g., tie strength), metaperception and other psychological variables related to the recommendation, and program-related variables (e.g., attractiveness of program).

In order to examine our hypotheses empirically, we matched CRM data from a large European consumer bank's with consumers' survey responses. The research context is the RRP of a large European consumer bank. Respondents were 300 inductors and the 300 inductees they had introduced to the bank. We added a control group of 300 customers who had never participated in the RRP, neither as inductors nor as inductees. Respondents were interviewed using a telephone survey conducted by a professional market research firm. Our analysis will generate important theoretical and managerial implications.
\end{abstract}

References available upon request 